\title{
Clinical Trial and Disease Search with Ad Hoc Interactive Ontology Alignments
}

\author{
Daniel Sonntag ${ }^{1}$, Jochen Setz ${ }^{1}$, Maha Ahmed-Baker ${ }^{1}$, and Sonja Zillner ${ }^{2}$ \\ 1 German Research Center for AI (DFKI) \\ Stuhlsatzenhausweg 3, 66123 Saarbruecken, Germany \\ 2 Siemens AG, Corporate Technology \\ Otto-Hahn-Ring 6, 81739 Munich, Germany
}

\begin{abstract}
We will explain how an LODD application based on diseases, drugs, and clinical trials can be used to improve the (ontology-based) clinical reporting process while, at the same time, it improves the patient follow-up treatment process. Specific requirements of the radiology domain let us aggregate RDF results from several LODD sources such as DrugBank, Diseasome, DailyMed, and LinkedCT. The idea is to use state-of-the-art string matching algorithms which allow for a ranked list of candidates and confidences of the approximation of the distance between two diseases at query time. Context information must be provided by the clinician who decides on the "related"-mappings of patient context and links he wants to follow in order to retrieve disease and medication information.
\end{abstract}

\section{Introduction}

In many industrial domains such as medical radiology, a vast amount of images is produced and the medical image annotations must be refined and augmented during a complex medical workflow. We use several technologies for the semantic annotation of medical images and radiology reports [10. The problem is that these annotations only capture descriptive information, i.e., the clinical observations, the various identified symptoms, and the discovered findings. But in practice, clinicians often want to search for higher level information such as interventions and the respective side effects, or associated information such as related drugs and diseases in the context of identified symptoms, etc. As a matter of fact, expert knowledge about diseases, drugs, and clinical trails is often not available through internet searches or too imprecise.

Our experiences throughout the THESEUS MEDICO11 and RadSpeech 2 research projects (which focus on semantic medical image search and user interaction [12, respectively) have shown us that several types of knowledge contained in Linked Data are relevant for the annotation of the images. In other words, when radiologists (or other clinicians) examine their patients' medical

\footnotetext{
${ }^{1}$ http://theseus-programm.de/en/920.php

${ }^{2}$ http://www.dfki.de/RadSpeech/ 
images they would additionally like to know whether previous diagnoses exist, if there has been a change in the case, and what kind of medication and treatment plan is foreseen. This requires the medical images to be annotated accordingly so that the radiologists can obtain all the necessary information starting with a computer tomography (CT) or magnet resonance (MR) image, and the case description in form of a patient record. We will explain how an LODD (http://www.w3.org/wiki/HCLSIG/LODD) application based on diseases, drugs, and clinical trials can be used to improve the (ontology-based) clinical reporting process while, at the same time improving the patient follow-up treatment process (i.e., monitoring the patient's health condition and the development of the disease). We will focus on the essential part of ontology matching between the medical reference ontology, Radlex 3 [5, and the available and relevant LODD data contained in DrugBank, DailyMed, and Diseasome which are mediated through the LinkedCT resources. LinkedCT, see http://linkedct.org, aims at publishing the first open Semantic Web data source for clinical trials data; it contains more than 60,000 trails, 14,243 conditions, and 67,271 interventions.

Essentially, the important mapping task between LinkedCT and Diseasome must be seen in the context of a more complex medical workflow which we will explain in detail. In addition, the mapping of several additional resources can only be done interactively, at query time, to meet both the data and the intentions of the clinician who searches for trail and drug information. The reason for this is that a radiologist can only decide in an ad hoc fashion whether two proposed "equality" or "related" matches are appropriate in a specific patient and knowledge retrieval context. This paper is structured as follows. Section 2 describes the clinical problem statement and argues in favour of a context-based interactive approach; section 3 describes the workflow we created in order to meet the clinical requirements while embedding the context-based interactive approach into a concrete knowledge retrieval scenario. Section 5 provides a first evaluation of the approach to meet the clinical requirements; section 6 concludes.

\section{Clinical Problem Statement}

In our carrier project THESEUS MEDICO, we envision a flexible and generic image understanding software. Semantics of the images plays the major role for access and retrieval. The next generation of intelligent, scalable, and robust search engines for the medical imaging domain should be based on semantic technologies. With the incorporation of higher level knowledge represented in ontologies, different semantic views of the same medical images (such as structural aspects, functional aspects, and disease aspects) can be explicitly stated and integrated.

\footnotetext{
${ }^{3}$ Radlex is a controlled vocabulary developed and maintained by the Radiological Society of North America (RSNA) for the purpose of indexing and retrieving radiology images and related information. Radlex contains 11,962 domain related terms (e.g., anatomy pathology or imaging techniques). Synonym information is partially indicated, such as Schatzki ring and Lower esophageal mucosal ring.
} 
A first analysis of the envisioned search functionality revealed that the association between observed diseases (e.g., lymphoma) and different, related types of the same disease serve as a valuable knowledge resource (as it can be used for refining the search query) when searching for similar patients and/or clinical trials. We identified related LODD resources to capture valuable associations between the various high-level concepts such as diseases, interventions, medications, symptoms, etc. Those concepts occur within the clinical diagnostic process and are thus very relevant for defining search queries. The two related LODD resources have been identified, Drugbank 4 , Diseasome 5 , and DailyMed 6 . Figure 1 shows the identified LODD resources and a potential interlinking.

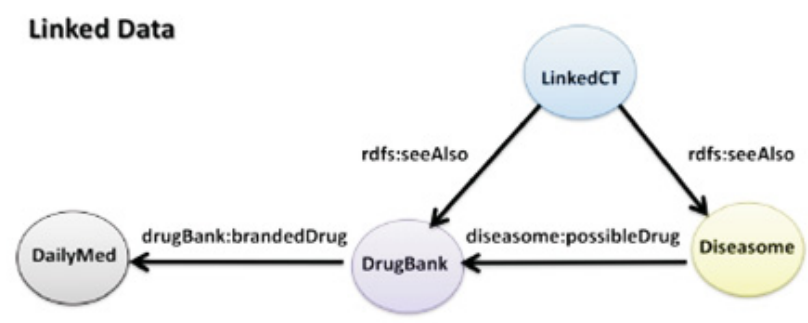

Fig. 1. Identified LODD resources

Existing medical ontologies for anatomy and disease related aspects (cf. FMA, RadLex or NCI-Thesaurus) usually focus on one particular domain, such as anatomy or radiology, and do not cover relations that link concepts from other domains such as those which link associated findings with diseases. Most medical ontologies of this scale for anatomy, disease, or drug aspects can be summarised as: (a) they are very large models, (b) they have extensive is-a hierarchies up to ten thousands of classes which are organised according to different views, (c) they have complex relationships in which classes are connected by a number of different relations, (d) their terminologies are rather stable (especially for anatomy) in that they should not differ too much in the different ontologies

${ }^{4}$ DrugBank is a large repository of small molecule and biotech drugs that contains detailed information about drugs including chemical, pharmacological, and pharmaceutical data in addition to sequence, structure, and pathway information. The Linked Data DrugBank contains 1,153,000 triples and 60,300 links.

${ }^{5}$ Diseasome contains information about 4,300 disorders and disease genes linked by known disorder's gene associations. It also indicates the common genetic origin of many diseases. The list of disorders, disease genes, and associations between them comes from the Online Mendelian Inheritance in Man (OMIM), which is a compilation of human disease genes and phenotypes. The Linked Data Diseasome contains 88,000 triples and 23,000 links.

${ }^{6}$ Dailymed provides up-to-date information about marketed drugs. Human Prescription Labels, OTC Labels, and Homeopathic Labels sum up to several million entries. 
(we will show the opposite for the cancer disease parts), and (e) their modelling principles are well defined and documented.

A variety of methods for ontology alignment have been proposed [13 2 4 1186 . The objective of the state-of-the-art in ontology mapping research includes the development of scalable methods (e.g., by combining very efficient string-based methods with more complex structural methods), and tools for supporting users to tackle the interoperability problem between distributed knowledge sources (e.g., editors for iterative, semi-automatic mapping with advanced incremental visualisations 9]). In addition, cognitive support frameworks for ontology mapping really involve users [3], or try to model a natural language dialogue for interactive semantic mediation [11.

One of the ontology matching problems in medicine is still that, in many cases, complex ontology matching algorithms cannot be used because they do not scale to sizes of medical ontologies - complex methods for ontology alignment in the medical domain turned out to be unfeasible because the concept and relation matrix is often on the scale of $100000 \times 100000$ alignment cells and appropriate subontologies cannot be created with state-of-the-art methods because of complex inter-dependencies.

Another problem is that when using those methods, we can only work with static mapping as a result of an offline matching process in which the mappings are independent of the context in which they are used. In the context of our medical use case, however, we learned in discussions with our clinicians that establishing clinical relevant associations between given clinical concepts has the potential to improve the search functionality. But that comes with the condition of a context-dependent quality and relevance of established associations (i.e., alignments) between clinical concepts which determines to which extent the search functionality can be improved.

We argued in 14 that annotating medical images with information available from LODD can eventually improve their search and navigation through additional semantic links. One outcome of our ontology engineering methodology 1513. was the semi-automatic alignment between radiology-related OWL ontologies (FMA 7 , and Radlex). This alignment could be used to provide new connections in the medicine-related linked data cloud. The fact that contextdependency may play a pivotal role for static alignments from FMA to Radlex was shown in 16. Why should this problem be more severe in the context of an online information retrieval task where diseases from LinkedCT and Diseasome have to be aligned?

Basically speaking, small changes in the nomenclature can make a big differences in the adequacy of the proposed mappings. This is driven by the fact that, in medicine, usually a very specific difference in the concept names might make a huge difference for their interpretation (therefore we should not even try to map LinkedCT and Diseasesome unless there are exact matches.) But the absence of globally unique identifiers for diseases (the URIs) forces us to provide the mappings. Instead of trying to infer such mappings on the large scale for disease data sources (LinkedCT, for example, has only 830 owl:sameAs links to 
Diseasome but contains more than 4600 different disease URIs), we try to establish an ad hoc mapping. Further, we ask whether static nton mappings between two medical ontologies are really necessary and represent really what is desired in a specific query situation. In the following, we will argue that, at least in our medical usecase, a rather different set of requirements exists.

For example, a clinical expert identifies patient cancer cells in the imaging data and is sure that they are from type "lymphoma". Hence our (Radlex) search term is "lymphoma". In order to decide on the follow-up treatment steps of the patient, he wants to search for similar patients/trials where similar patients have been successfully treated (case-based reasoning). Lymphoma diseases of patients can, however, be distinguished along three orthogonal dimensions:

1. the type (e.g., Hodgkin and non-Hodgkins lymphoma);

2. the stage (e.g., stage I to stage IV); and

3. the grade (e.g., low, intermediate or high grade).

As lymphomas of different type, stage, and grade grow at different rates, they respond differently to specific treatments. For that reason, clinicians need to know the particular type, stage, and grade of a patient's lymphoma for an adequate treatment. Accordingly, we have to filter out the relevant trails, or in other words, align the trials according to this complex information background.

For that reason, we cannot rely on semantic/structured-based knowledge models to establish associations for "fine-tuning" our search space. To the best of our knowledge, no formal knowledge structure exists that relies these three dimension (type, stage, and grade) in a proper manner/model.

For the same reason, we also did not use a semantic similarity measure. Initially, it might appear appropriate to use abbreviation lists, synonym sets, etc. Although it seems to be obvious that purely syntactic (approximate) string matching techniques are not sufficient to deal with different data representations, different linguistic surface forms, and missing information about type, stage, and grade, it is wrong to believe that the potential increase in recall when using such query expansion only means a little loss of precision.

However, our interactive workflow is designed to increase precision at a stable recall level as our examples from LinkedCT and Diseasome will show. (The recall level can, however, be controlled by declaring the thresholds for individual ad hoc matchers.)

\section{Proposed Workflow}

Figure 2 shows the proposed workflow.

1. The user inputs a medical term or refers to the existing CT or MR image annotation. In both cases, a Radlex term is derived and used as the "disease_name". 
2. MEDICO then executes a SPARQL query that includes an (approximate) string matching filter to LinkedCT. (We made positive experience with the SPARQL operator implemented in Virtuoso (filter(regex(?LCT_diseases_name, "*disease_name*", " $i$ ")). (This accounts for the online processing demand as well as the user's expectation of the LinkedCT's "first results".)

3. The user browses the retrieved trails with a facetted browsing functionality we implemented (see section 5) and selects the trails he is interested in.

4. Then, he selects a disease from a specific trail.

5. The system now either follows the link to Diseasome (trivial case) or invokes the interactive, approximate string-based matching procedure when there is no link available.

6. According to the found disease-URIs and the de-referenced possible drugs from DrugBank, the user can click further to obtain the highly desired DailyMed contents.

This process is utterly transparent to the user for two reasons. First, during the direct-manipulation facetted browsing and search interface, he knows exactly at which stage he is and exactly when additional input from him is required (stage 5 ). Second, built on the mental model of the retrieval stages and the context knowledge he has gained by inspecting the patient file, reflecting on the Radlex term, and following only the disease links he is interested in, the clinician knows how to interpret the proposed mappings. It becomes clear that the clinician is not necessarily interested in pure equality (or subsumption mapping), but uses a more underspecified "related-to" mapping/alignment. Interestingly, this "related-to" mapping only makes sense in this dynamic retrieval process where he or she uses the proposed mappings as a further anchor point in his extended search context. In other words, the user defines what he means by the mappings in an ad hoc fashion. Therefore, we cannot use static mappings at all or record our mappings as static ones. The question is whether this procedure really enhances the robustness of the overall search interface in specific, usecase-relevant retrieval situations (cf. section 5).

\section{String-Based Interactive Matching Approach}

The idea is to use state-of-the-art string matching algorithms which allow for a ranked list of candidates and confidences of the approximation of the distances between two strings. In addition, the individual results must be homogeneous in the sense that they are comparable and can be combined. The combination of the results of the string matching approaches is essential because the variety of similarity functions that exist in literature have specific positive but also negative characteristics that have to be taken into account and used in the right way. First, we define the string matching and the under context filter functions. 


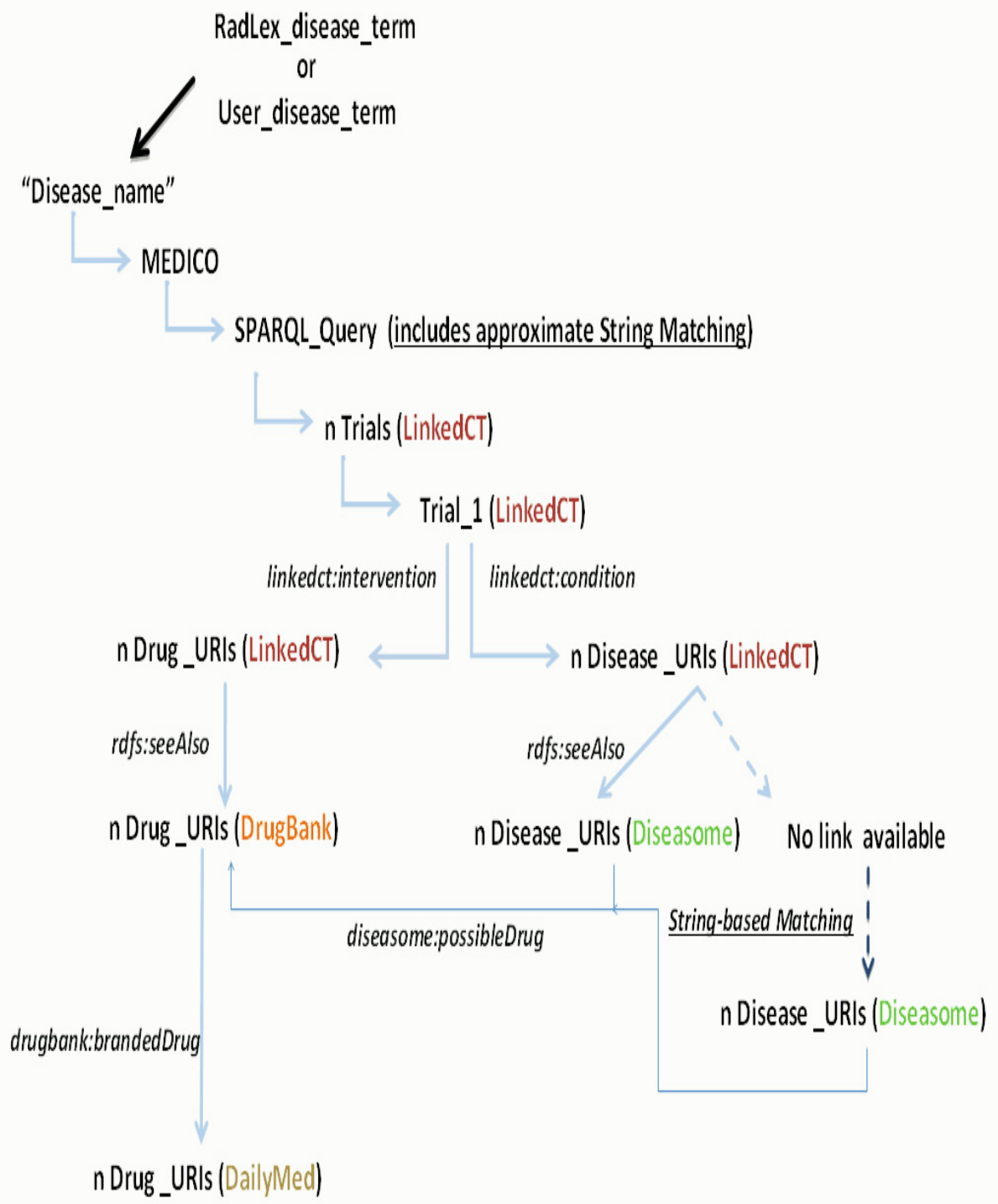

Fig. 2. Workflow of the Clinical Information Retrieval and Ontology Matching Tasks 
Definition 1 (String Matching Function). Given a input search term $t$ and a LODD knowledge repository $L$, be $\pi$ a string matching filter function $\pi: t \times L \longrightarrow\{(t, l) \mid l \in L \wedge \sigma(t, l)\}$, with $\sigma$ a boolean similarity function $s$ that is true whenever the terms $t$ and $l$ yield a string similarity according to the established similarity measure.

Definition 2 (User Context Filter Function). Given a input search term $t, S$ a set of terms, then we define a user context filter function

$\pi_{\text {user }}: t \times S \longrightarrow\left\{(t, s) \mid s \in S \wedge \sigma_{\text {relevant }}(t, s)\right\}$,

with $\sigma_{\text {relevant }}$ being a boolean function that is true whenever the similarity of the terms $t$ and $s$ is relevant in the context of the user.

Function $\pi$ let's us define a threshold on the individual contributors to an aggregate measure of string matches we take into account for the interaction step; $\pi_{\text {user }}$ allows us to formalise the selection step the clinician performs according to his context information. If $\pi_{u s e r}=t r u e$, we established an ad hoc "related-to" mapping.

We used the similarity measures $\operatorname{sim}_{W \text { Jaccard }}$, sim $_{W \text { Jaro }}$, sim $_{W \text { Jaro-Winkler }}$, and sim $_{e d}$. and aggregated their results to a "star recommendation" scheme starsTotal, whereby starsTotal $=\sum_{i=1}^{4} \pi:\left(\sigma=\operatorname{sim}_{i}\right)$ The individual measures are defined as follows.

Formally, the Weighted Jaccard measure takes two disease names $d_{1}, d_{2}$ and computes (only) the fraction of tokens that are present in both multi-word terms.

$$
\begin{gathered}
\operatorname{sim}_{W \text { Jaccard }}\left(d_{1}, d_{2}\right)=\frac{\sum_{t \in \mathbf{d}_{\mathbf{1}} \cap \mathbf{d}_{\mathbf{2}}} w(t, R)}{\sum_{t \in \mathbf{d}_{\mathbf{1}} \cup \mathbf{d}_{2}} w(t, R)} \\
\operatorname{sim}_{W \text { Jaro }}\left(d_{1}, d_{2}\right)=\frac{1}{3} *\left(\frac{\left|d_{1}^{\prime}\right|}{\left|d_{1}\right|}+\frac{\left|T_{d_{1}^{\prime}, d_{2}^{\prime}}\right|}{\left|d_{2}\right|}+\frac{\left|d_{1}^{\prime}\right|}{\mid}\right)
\end{gathered}
$$

The Jaro-Winkler variant of the Jaro measure takes the longest common prefix of $d_{1}$ and $d_{2}$ as length $\mathrm{P}$ to compute an edit distance. Letting $P^{\prime}=\max (P, 4)$, the Jaro-Winkler distance is defined by

$$
\operatorname{sim}_{W \text { Jaro-Winkler }}\left(d_{1}, d_{2}\right)=\operatorname{sim}_{W J a r o}\left(d_{1}, d_{2}\right)+\frac{P^{\prime}}{10} *\left(1-\operatorname{sim}_{W J a r o}\left(d_{1}, d_{2}\right)\right) \text {. }
$$

The Damerau-Levenshtein Algorithm is implemented as follows: we use the function $\operatorname{sim}_{e d}: \mathbb{N} \times \mathbb{N} \rightarrow \mathbb{N}$ taking $i=\left|d_{1}\right|$ and $j=\left|d_{2}\right|$ where $i, j \mapsto x$ is defined by:

$$
\begin{aligned}
\operatorname{sim}_{e d}(0,0)= & 0 \\
\operatorname{sim}_{e d}(i, 0)= & i \\
\operatorname{sim}_{e d}(0, j)= & j \\
\operatorname{sim}_{e d}(i, j)= & \min \left\{\operatorname{sim}_{e d}(i-1, j)+1, \operatorname{sim}_{e d}(i, j-1)+1,\right. \\
& \operatorname{sim}_{e d}(i-1, j-1)+d\left(d 1_{i}, d 2_{j}\right), \\
& \left.\operatorname{sim}_{e d}(i-2, j-2)+d\left(d 1_{i}, d 2_{j-1}\right)+d\left(d 1_{i-1}, d 2_{j}\right)+1\right\},
\end{aligned}
$$

whereby the function $d\left(d 1_{i}, d 2_{j}\right)$ counts the distance between two letters. 
The more measures we have that agree on two (disease) terms as being "related", the more stars are visible as our recommendation for the clinician. However, the decision whether the recommendation is in line with the user's expectations is taken by the clinician and includes the outcome of $\pi_{\text {user }}$.

For example, n-gram measures such as Weighted Jaccard make it possible to measure the similarity based on specific words and ignore others which are expected to be unimportant. But how should we know which tokens are unimportant? Formally, the Weighted Jaccard measure takes two disease names $d_{1}, d_{2}$ and computes (only) the fraction of tokens that are present in both multi-word terms.

\section{Evaluation}

We evaluated the results of our individual similarity measures and found some special characteristics of the measures when applied to our specific data. The Weighted Jaccard method is useful to crop off stop words like "disease" as in "Castleman's Disease" to map against "Castleman", but is completely useless for, e.g., the stages of a disease case, a factor extremely important for lymphoma cases. This outcome reveals that when using this measure for medical linked data, it would be based on the wrong assumption that medical concept names contain negligible tokens. But our staging information and related type information is coded in those tokens. For example, the LinkedCT terms "Lymphocytic Leukemia, L1" and "Lymphocytic Leukemia, L2" differ only in the stage number, but this information is essential for finding related trials and drugs.

But also the traditional string similarity measures such as $\operatorname{sim}_{e d}$ cannot be used without caution. For example, in [16] we emphasised (in the context of the FMA ontology) that it contains closely related concepts such as "Anterior cervical lymph node" and "Set of anterior cervical lymph nodes" which could not be identified as duplicates with the $\operatorname{sim}_{e d}$ function. The variation of the linguistic surface forms might vary even to a greater extend when taking multiple ontologies (i.e., LinkedCT and Diseasome) into account. On the other hand, $\operatorname{sim}_{e d}$ works very well in identifying related staging cases when only the stage numbers or similar sub-expressions are different (cf . the "Lymphocytic Leukemia L1 / L2").

As a result of the observation that several distance functions have different performances depending on the characteristics of the data (such as length of the string, token permutations, etc.) we evaluated our ensemble measure with the "star" recommendations. Since the functions are independently applied to the disease names $d_{1}, d_{2}$ and aggregated into a combined measure by specifying the thresholds and weights of the individual distance function calls, a vast improvement in the robustness could be achieved. A closer examination of the disease names stored in LinkedCT and Diseasome provides an explanation: many disease name contain long digit sequences, for instance 'G09330582163324' (LinkedCT). In most cases the digits refer to important type, stage, and grade 


\begin{tabular}{|l|l|l|l|l|l|}
\multicolumn{2}{l}{ Lymphoma, Mantle cell (LinkedCT) } \\
\hline & Disease Name (Diseasome) & Trivial ED & Tr ED Weighted & Jaccard & Jaro Winkler \\
\hline$(1)$ & Lymphoma, mantle cell & 1 & 1 & 0.076 & 0.031 \\
\hline$(2)$ & Lymphoma, T-cell & 5 & 5 & 0.076 & 0.121 \\
\hline$(3)$ & Lymphoma, MALT & 8 & 8 & 0.037 & 0.134 \\
\hline$(4)$ & Lymphoma, somatic & 9 & 9 & 0.333 & 0.169 \\
\hline$(5)$ & Leukemia, acute myelogenous & 1000 (max) & 1000 (max) & 0.444 & 1000 (max)
\end{tabular}

Fig. 3. Mantle Cell Lymphoma as input from LinkedCT

\begin{tabular}{|l|l|l|l|}
\hline & Disease Name (Diseasome) & Quality & Select \\
\hline (1) & Lymphoma, mantle cell & & \\
\hline (2) & Lymphoma, T-cell & \\
\hline (3) & Lymphoma, MALT & \\
\hline (4) & Lymphoma, somatic & \\
\hline (5) & Leukemia, acute myelogenous &
\end{tabular}

Fig. 4. Mantle Cell - Choice box for the proposed and selected "related"-mappings to Diseasome

information which is covered by the combined measure. The type, stage, and grade information we finally optimised for, are the following:

1. the type of the disease: "Diabetes Mellitus, Type 1" , "Diabetes Mellitus, Type 2",

2. the stage of the disease: "Early Stage Breast Cancer (Stage 1-3)",

3. the age of the patient as an indirect type classification: "ICU Patients 18 Years or Older",

4. the date of the disease outbreak: "2009 H1N1 Influenza",

5. genetic information; location of deleted region in the chromosome: "22q11.2 Deletion Syndrome".

We then evaluated the interactive procedure in the context of our lymphoma case. The lymphoma case reveals that LinkedCT enumerates 459 lymphoma disease URIs (and Diseasome only 25 URIs) with the same name observations. Since either the LinkedCT term or the Diseasesome term often lacks context information, we can only rely on the interactive workflow because the context is provided by the context factors explained above - and which are accessible to the expert while focussing on the suggestions the system provides. Here is an example of the individual measure outcomes for "Mantle Cell Lymphoma" from LinkedCT (figure 3), for which no links to Diseasome exist.

The aggregated "star recommendation" is attached to the suggested choice box for the as hoc "related" mappings (figure 4). 


\begin{tabular}{|c|c|}
\hline \multicolumn{2}{|c|}{ Linkedct-Result: Trial NCT00723099 } \\
\hline $\begin{array}{l}\text { Endpoint Classilication: Efficacy Study, Intervention Model: } \\
\text { Purpose:Treatment (Interventional) }\end{array}$ & Single Group Assignment, Masking: Open Label, Primary \\
\hline $\begin{array}{l}\text { LinkedCT-Diseases: Stage IV Cutaneous T-cell } \\
\text { Non-Hodgkin Lymphoma, Stage III Cutaneous T- } \\
\text { cell Non-Hodgkin Lymphoma, Stage II Cutaneous } \\
\text { T-cell Non-Hodgkin Lymphoma, Stage I Cutaneous } \\
\text { T-cell Non-Hodgkin Lymphoma, and Recurrent } \\
\text { Cutaneous T-cell Non-Hodgkin Lymphoma }\end{array}$ & $\begin{array}{l}\text { LinkedCT-Drugs: allogeneic hematopoietic stem } \\
\text { cell transplantation, umbilical cord blood } \\
\text { transplantation, total-body irradiation, } \\
\text { mycophenolate mofetil, cyclosporine, } \\
\text { cyclophosphamide, and fludarabine phosphate }\end{array}$ \\
\hline Diseasome-Diseases: & $\begin{array}{l}\text { Dailymed-Drugs: DOXYCYCLINE HYCLATE, } \\
\text { Mycophenolate Mofetil, Cyclosporine, } \\
\text { Cyclophosphamide, Sodium Chloride, and } \\
\text { Fludarabine Phosphate }\end{array}$ \\
\hline (October 2010) & Fred Hutchinson Cancer Research Center \\
\hline \multicolumn{2}{|c|}{ Linkedct-Result: Trial NCT00808171 } \\
\hline \multicolumn{2}{|c|}{$\begin{array}{l}\text { Allocation: Randomized, Endipoint Classification: Salety/Elficacy Study, Intervention Model: Parallel Assignment, } \\
\text { Masking: Double Blind (Subject,Caregiver, Investigator, Outcomes Assessor), Primary Purpose: Supportive Care } \\
\text { (Interventional) }\end{array}$} \\
\hline LinkedCT-Diseases: Non-Hodgkin Lymphoma & LinkedCT-Drugs: nitrous oxide \\
\hline Diseasome-Diseases: Non-Hodgkin lymphoma & Dailymed-Drugs: \\
\hline (December 2008) & Federal University of Minas Gerais \\
\hline
\end{tabular}

Fig. 5. Results of the Trial search

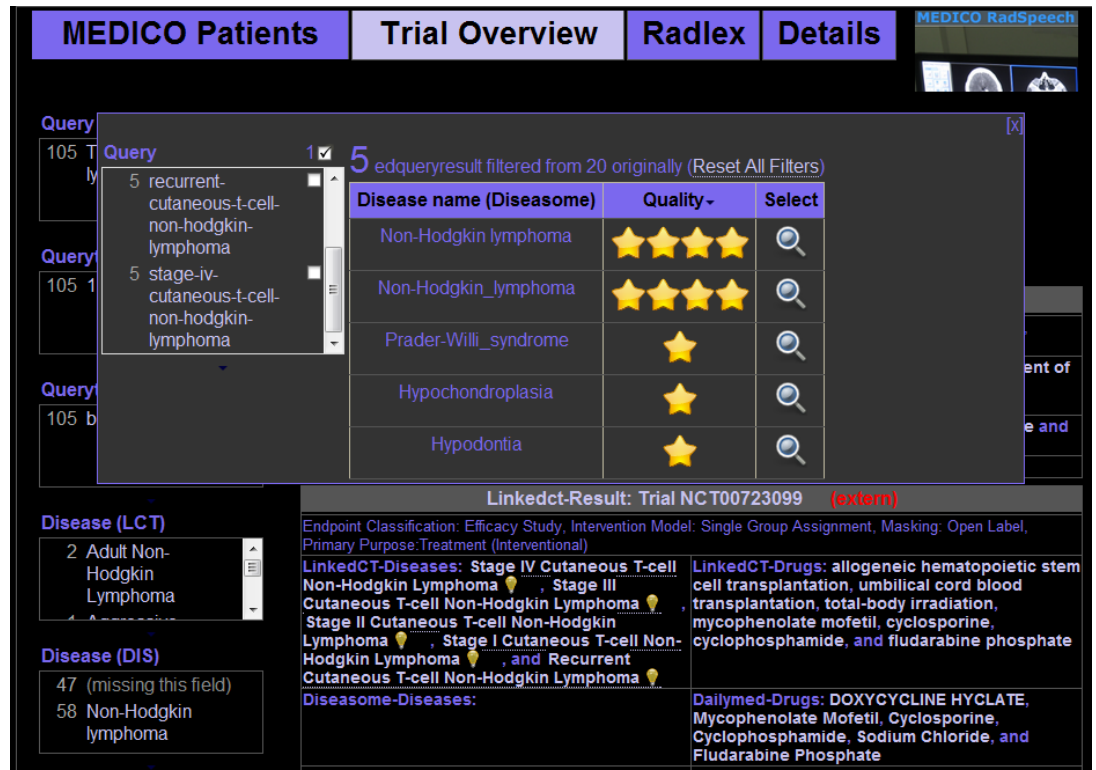

Fig. 6. Display and Selection of the Proposed ad hoc Mappings

The choice box is being displayed within the facetted browsing tool. Our tool allows a user to filter thousands of results according to the best-ranked string matches and linked data relations in a convenient way. Clinician can retrieve the trails they might be interested in in just a few seconds. We implemented the graphical user interface by using open-source knowledge management tools 
such as Exhibit, see http://www.simile-widgets.org/exhibit/. Figure 5] shows the embedded iPad GUI widget at stage 4 of the proposed workflow. The circle below indicates the trivial matching case - an equality link was provided by LinkedCT. The circle above shows the interesting case, the Diseasome disease is unknown. A click on a particular Stage I, II, or III lymphoma case evokes the ad hoc similarity search which results in the interactive mapping suggestion displayed in figure 6 .

\section{Conclusion}

We explained how an LODD application based on diseases, drugs, and clinical trials can be used to improve the clinical reporting process. In order to get information about trails, drugs, and diseases, several LODD sources can be addressed and the contained knowledge can be combined. The clinical problem statement suggested that in order to make the application useful for improving the patient follow-up treatment process, specific non-existing mappings must be provided. The important mapping task lies between LinkedCT and Diseasome in the context of a more complex medical workflow which we developed. We argued that ad hoc interactive string-based ontology alignments should be used to propose several ad hoc mappings to the user. The user can then verify them at the knowledge retrieval stage while using the facetted browsing tool which implements the graphical user interface of the proposed workflow. An evaluation has shown that the interactive approach is useful and that underspecified "related" mappings are often more useful than precise "equality" mappings in the medical domain. In general, the discovery of such "related" links which are typed, dynamic, and bound to a specific user, data, and application context, should be an active research area.

Acknowledgements. We would like thank all colleagues at DFKI and all partners in the MEDICO and RadSpeech projects for their valuable contributions to the iterative process described here. This research has been supported by the THESEUS Programme funded by the German Federal Ministry of Economics and Technology (01MQ07016).

\section{References}

1. Doan, A., Madhavan, J., Domingos, P., Halevy, A.: Ontology matching: A machine learning approach. In: Handbook on Ontologies in Information Systems, pp. 397-416. Springer (2003)

2. Euzenat, J., Shvaiko, P.: Ontology matching. Springer, Heidelberg (2007)

3. Falconer, S.M., Noy, N., Storey, M.-A.D.: Towards understanding the needs of cognitive support for ontology mapping, vol. 225 (2006)

4. Kalfoglou, Y., Schorlemmer, W.M.: Ontology mapping: The state of the art. In: Semantic Interoperability and Integration (2005)

5. Langlotz, C.P.: Radlex: A new method for indexing online educational materials. RadioGraphics 26, 1595-1597 (2006) 
6. Noy, N.F.: Tools for mapping and merging ontologies. In: Handbook on Ontologies, pp. 365-384 (2004)

7. Noy, N.F., Rubin, D.L.: Translating the foundational model of anatomy into OWL. Web Semant. 6, 133-136 (2008)

8. Rahm, E., Bernstein, P.A.: A survey of approaches to automatic schema matching. The VLDB Journal 10, 334-350 (2001)

9. Robertson, G.G., Czerwinski, M.P., Churchill, J.E.: Visualization of mappings between schemas. In: CHI 2005: Proceedings of the SIGCHI Conference on Human Factors in Computing Systems, pp. 431-439. ACM, New York (2005)

10. Seifert, S., Kelm, M., Möller, M., Mukherjee, S., Cavallaro, A., Huber, M., Comaniciu, D.: Semantic annotation of medical images. In: Proceedings of SPIE Medical Imaging, San Diego, CA, USA (2010)

11. Sonntag, D.: Embedded benchmarking and expert authoring for ontology mapping and alignment generation. In: Proceedings of the Fifth International Conference on Formal Ontology in Information Systems, FOIS (2008)

12. Sonntag, D., Schulz, C., Reuschling, C., Galarraga, L.: Radspeech, a mobile dialogue system for radiologists. In: Proceedings of the International Conference on Intelligent User Interfaces, IUI (2012)

13. Sonntag, D., Wennerberg, P., Buitelaar, P., Zillner, S.: Pillars of Ontology Treatment in the Medical Domain. In: Cases on Semantic Interoperability for Information Systems Integration: Practices and Applications, pp. 162-186. Information Science Reference (2010)

14. Sonntag, D., Wennerberg, P., Zillner, S.: Applications of an ontology engineering methodology. AAAI Spring Symposium Series (2010)

15. Wennerberg, P., Zillner, S., Möller, M., Buitelaar, P., Sintek, M.: KEMM: A Knowledge Engineering Methodology in the Medical Domain. In: Proceedings of the 2008 Conference on Formal Ontology in Information Systems, pp. 79-91. IOS Press, Amsterdam (2008)

16. Zillner, S., Sonntag, D.: Aligning medical ontologies by axiomatic models, corpus linguistic syntactic rules and context information. In: Proceedings of the 24th International Symposium on Computer-based Medical Systems, CMBS (2011) 